\title{
Impacto de una intervención educativa en escolares y madres de familia para la disminución de sobrepeso
}

\author{
Martha Lilia Bernal Becerril,* Gandhy Ponce Gómez,* Sandra M Sotomayor Sánchez,* Beatriz Carmona Mejía**
}

\begin{abstract}
RESUMEN
Introducción: La nutrición tiene un papel de gran importancia en la salud; la obesidad es resultado de un desequilibrio entre la ingestión y el gasto energético. En México (2006), la prevalencia nacional combinada de sobrepeso y obesidad en niños de 5 a 11 años fue de alrededor del 26\% para ambos sexos. Objetivo: Medir el impacto de una intervención educativa para disminuir el sobrepeso con base en los estilos de vida en una población de escolares de 6 a 11 años de edad. Metodología: Considerando el grave problema de salud que representa el sobrepeso y la obesidad en la población escolar, se propone este estudio cuasiexperimental, longitudinal correlacional y comparativo. Resultados: El pre-test arrojó que el nivel de conocimientos de madres y niños sobre la nutrición era insuficiente; los estilos de vida saludables se hallaron en nivel óptimo para las madres y suficiente para los niños, sin detectarse diferencias estadísticamente significativas entre el grupo experimental y el grupo control. Posterior a la intervención, existieron diferencias significativas entre el grupo experimental de madres, aumentando los conocimientos y mejorando las actitudes, mientras que en los niños no hubo diferencias significativas. Discusión y conclusiones: Se requiere complementar la actividad física con hábitos alimenticios adecuados, reconociendo la necesidad de un trabajo multidisciplinario y la participación activa de la sociedad.
\end{abstract}

Palabras clave: Intervención educativa, estilos de vida, hábitos alimenticios adecuados.

\section{Impact of an educational intervention in school and mothers for reducing overweight}

\begin{abstract}
Introduction: Nutrition is one of the principal determinant of health; obesity is the result of an imbalance between intake and energy expenditure. In Mexico (2006), the combined national prevalence of overweight and obesity in children aged 5 to 11 years, was around $26 \%$ for both sexes. Objective: To measure the impact of an educational intervention to reduce overweight based on the lifestyles of a population of schoolchildren aged 6 to 11 years old. Methodology: Considering the serious health problem of overweight and obesity in school children is that it is proposed to study quasi-experimental, correlational and comparative longitudinal. Results: The pre-test showed that the level of knowledge of mothers and children, about of nutrition, was at insufficient levels, and lifestyles, were found at optimum level for mothers and children sufficient. No statistically significant differences between experimental and control group. After the intervention there were significant differences between experimental group of mothers by increasing knowledge and attitudes, while the children did not differ significantly by group. Discussion and conclusions: We require complementary efforts to food and nutrition on the incorporation of physical activity and proper dietary habits, recognizing the need for a multidisciplinary approach and active participation in society.
\end{abstract}

Key words: Educational intervention, lifestyle, proper eating habits.

* Profesor de Carrera Asociado C, ENEO-UNAM.

** Profesor de Carrera Asociado C, FES Zaragoza-UNAM.

Correspondencia: Mtra. Martha Lilia Bernal Becerril

E-mail: marthaliliabb@hotmail.com

Este artículo puede ser consultado en versión completa en http://www.medigraphic.com/enfermerianeurologica 


\section{INTRODUCCIÓN}

L a nutrición, a lo largo de la vida, es una de las principales determinantes de la salud, del desempeño físico y mental y de la productividad. La mala nutrición (tanto la desnutrición como el exceso en la ingesta) tiene causas complejas que involucran determinantes biológicos, socioeconómicos y culturales.

El problema de la obesidad es resultado de un desequilibrio entre la ingestión y el gasto energético; particularmente es consecuencia de la ingestión de dietas con alta densidad energética y bajas en fibra y de bebidas azucaradas, en combinación con una escasa actividad física. Esta última se ha asociado a la urbanización, al crecimiento económico y a los cambios en la tecnología para la producción de bienes y servicios, así como en los estilos de vida y de recreación. ${ }^{1}$ De acuerdo con la Organización Mundial de la Salud, la obesidad ha alcanzado proporciones epidémicas a nivel mundial y cada año mueren -como mínimo- 2.6 millones de personas a causa de la obesidad o el sobrepeso. Aunque anteriormente se consideraba un problema confinado a los países de altos ingresos, en la actualidad la obesidad también es prevalente en los países de ingresos bajos y medianos. ${ }^{2}$ En 2010, alrededor de 43 millones de niños menores de cinco años de edad tenían sobrepeso. En los países en desarrollo, están viviendo cerca de 35 millones de niños con sobrepeso, mientras que en los países desarrollados esa cifra es de 8 millones. ${ }^{3}$

En México, de acuerdo con la Encuesta Nacional de Salud realizada en 2006, se muestra que la prevalencia nacional combinada de sobrepeso y obesidad en niños de 5 a 11 años, utilizando los criterios de la International Obesity Task Force (IOTF), es de alrededor de $26 \%$ para ambos sexos, $26.8 \%$ en niñas y $25.9 \%$ en niños, lo que representa alrededor de 4,158,800 escolares en el ámbito nacional con sobrepeso u obesidad. ${ }^{4}$

Actualmente, México es el primer país en Latinoamérica con alta incidencia de obesidad en escolares, condición que se ha incrementado de manera impresionante.

Según un comunicado de prensa del 2006 emitido por la Secretaría de Salud, el Servicio de Endocrinología Pediátrica del Hospital Infantil de México "Federico Gómez" declaró que el $40 \%$ de la población infantil en nuestro país sufre sobrepeso y obesidad. Esto se debe en gran parte a la carga genética de los mexicanos que predispone al sobrepeso. Además, el cambio de vida que ha tenido la sociedad tanto en sus hábitos alimenticios como de actividad física es un factor de gran importancia. ${ }^{5}$

Los niños con obesidad pueden sufrir de hipertensión, colesterol elevado y resistencia a la insulina desde la infancia y la pubertad, y continúan con el riesgo en la etapa adulta.
En varones, aumenta el riesgo de aterosclerosis, infartos al miocardio, accidentes vasculares cerebrales, diabetes, así como cáncer de colon. Las mujeres, en cambio, son proclives a padecer artritis degenerativa, aumento de la presión arterial en el embarazo y predisposición de fracturas de cadera. Especialistas del Instituto Nacional de Pediatría estiman que niños con sobrepeso de $25 \%$ adicional a lo normal tienen mayor probabilidad de presentar alteraciones hormonales. En la mujer, el sobrepeso puede generar infertilidad, ovarios poliquísticos y alteraciones del ritmo menstrual. ${ }^{6}$

\section{OBJETIVO}

Medir el impacto de una intervención educativa para disminuir el sobrepeso con base en los estilos de vida de una población de escolares entre 6 y 11 años de edad de una escuela primaria en el D.F.

\section{ANTECEDENTES}

Diversos estudios locales, regionales e internacionales han estudiado el problema de salud de sobrepeso y obesidad en escolares como en otros grupos de edad de diferentes poblaciones y aquí damos cuenta de algunos de ellos.

Moraes, Beltrán, Mondini y Freitas, en un estudio realizado en México sobre la prevalencia de sobrepeso y obesidad y factores relacionados, señalan una prevalencia mayor para el sobrepeso que para la obesidad, reconociendo como factores de riesgo para el sobrepeso: la escolaridad de la madre, los hábitos de comer cuando están viendo la televisión, las actividades sedentarias y un peso al nacer mayor de $2.890 \mathrm{~g}$. En el Instituto Mexicano del Seguro Social, Ortiz, Rodríguez, Rurangriwa y Pérez ejecutaron un "Programa multidisciplinario de atención al niño obeso y con sobrepeso" a través de dieta, ejercicio, psicología para reducir el peso y normalización de los parámetros metabólicos en niños obesos y con sobrepeso. El estudio formó tres grupos: uno fue sometido a un programa de dieta balanceada, ejercicio y apoyo psicológico, otro a educación nutricional y el tercero fue de control, midiéndose los parámetros antropométricos y metabólicos al principio y a los cuatro meses de la intervención. Se obtuvo una $\mathrm{p}=0.05$, intervalo de confianza de $95 \%$. Los resultados arrojaron que hubo una reducción estadísticamente significativa en el peso, IMC, perímetro abdominal, presión arterial sistólica y diastólica en el grupo que recibió el programa multidisciplinario, y sin cambios estadísticamente significativos en el grupo de nutrición y en el de control. Se concluyó que la educación nutricional sin la complementación con un cambio en la actividad física no conlleva resultados óptimos, reconociendo la necesidad 
de un trabajo multidisciplinario y la participación activa de la sociedad en general. ${ }^{8}$

Francisco Moraga, María Jesús Rebollo, Pamela Bórquez, Jeannette Cáceres y Carlos Castillo realizaron un estudio denominado Tratamiento de la obesidad infantil: Factores pronósticos asociados a una respuesta favorable, ${ }^{9}$ en el cual pretendieron analizar los factores familiares, clínicos o personales que pudieran servir como predictores de éxito terapéutico en el control ambulatorio del niño obeso a través de un diseño prospectivo en 88 pacientes obesos de ambos sexos. Realizaron un interrogatorio, una evaluación antropométrica y una encuesta de actividad física e ingesta; solicitaron exámenes de laboratorio y efectuaron educación nutricional, alternando controles mensuales, grupales e individuales por nutricionista y médico. Los resultados reportaron que el $48 \%$ abandonó el control antes de los seis meses de seguimiento; de los que continuaron, $65 \%$ bajó de peso sobre 5\% de su IMC o P/T inicial (34\% del total). Encontraron hipercolesterolemia $<200 \mathrm{mg} / \mathrm{dL}$ en $15 \%$ de los niños e hipertensión, sin obtenerse asociación con otros factores estudiados: edad y educación materna, sexo, peso al nacer, tipo de familia, apreciación de obesidad y control espontáneo.

Briz Hidalgo FJ, Cos Blanco AI y Amate Garrido AM llevaron a cabo un estudio que titularon Prevalencia de obesidad infantil en Ceuta. Estudio PONCE 2005, ${ }^{10}$ el cual pretendió conocer la dimensión epidemiológica de la obesidad infantil en Ceuta, España, analizando a 514 niños en edad escolar y determinando el peso y la talla. Encontraron que la prevalencia de obesidad (IMC $\geq$ percentil 97) en niños de 6 a 13 años de edad se estima en un $8.75 \%$ y para el sobrepeso (IMC $\geq$ percentil 85 ) en un $13.81 \%$. En conjunto, sobrepeso y obesidad suponen el $22.57 \%$. La prevalencia de obesidad es más elevada en las mujeres (9.28\%) que en los varones $(8.50 \%)$, diferencia estadísticamente no significativa. En ninguno de los dos sexos se encontraron diferencias significativas según la edad.

\section{METODOLOGÍA}

Se trató de un estudio cuasiexperimental, longitudinal (se realizó un pre-test y un pos-test), correlacional y comparativo. Para la evaluación del impacto de una intervención educativa se formaron dos grupos: uno experimental y otro control, ambos constituidos de 20 madres y 20 niños cada uno, haciendo un total de 40 niños con sobrepeso u obesidad más las 40 madres correspondientes $\left(\mathrm{n}_{\text {niños }}=40 \mathrm{y} \mathrm{n}_{\text {madres }}=40\right)$. El instrumento de recolección de datos denominado $\mathrm{Co}$ nocimientos sobre una nutrición adecuada y estilos de vida saludables, elaborado para el caso y validado por cinco expertos en nutrición, cuenta con una sección de 20 ítems que indaga conocimientos relacionados con aspectos nutricionales con respuesta de opción múltiple donde sólo una es verdadera, y la sección de estilos de vida saludable, integrada por 20 ítems (escala Likert de 1 a 5 , donde 5 es siempre y 1 nunca), el cual, tras la recolección alcanzó un alfa de Cronbach de 0.876 , con lo que mostró ser válido y confiable para los fines de esta investigación. La ponderación que clasifica tanto el nivel de conocimientos como los estilos de vida saludables se muestra en el cuadro I.

La intervención educativa en la variable independiente se ejecutó de la siguiente forma: para el grupo control, los licenciados en enfermería con asesoría de un especialista en nutrición fueron los encargados de dar una plática educativa sobre hábitos saludables en la alimentación, la cual tuvo una hora de duración y se les entregó trípticos a las madres y a los niños.

Para el grupo experimental, previa estandarización del procedimiento con tres licenciados en enfermería y obstetricia, se organizaron y ejecutaron dos intervenciones educativas semanales durante tres meses, contando con la asesoría de un pedagogo, un psicólogo y un nutricionista, siendo un total de 24 sesiones al término de la jornada escolar (12:30 pm), las cuales tuvieron una duración aproximada de 30 minutos con temas como: causas y riesgos de la obesidad, hábitos alimenticios saludables, hábitos riesgosos de la alimentación inadecuada, consecuencias psicológicas, sociales y fisiológicas de la diabetes, grupos alimenticios y un taller de cocina donde se orientó acerca de la preparación de alimentos. Una vez concluida la intervención se aplicó un pos-test en el grupo experimental y en el grupo control.

\section{RESULTADOS}

Para los resultados del pre-test, se detectó que el nivel de conocimientos de madres y niños acerca de la nutrición era insuficiente, y respecto a los estilos de vida saludables se hallaron óptimos en las madres y suficientes en los niños, sin detectarse diferencias estadísticamente significativas entre grupo experimental y grupo control (Figura 1).

Cuadro I. Ponderación, nutrición y estilos de vida.

\begin{tabular}{|c|c|}
\hline Nivel de conocimientos & Ponderación \\
\hline Conocimientos muy óptimos acerca de la nutrición adecuada & $8.8-10.0$ \\
\hline Conocimientos óptimos acerca de la nutrición adecuada & 7.4-8.7 \\
\hline Conocimientos suficientes acerca de la nutrición adecuada & $6.01-7.3$ \\
\hline Conocimientos insuficientes acerca de la nutrición adecuada & $<6.01$ \\
\hline Estilos de vida saludables & Ponderación \\
\hline Estilos de vida muy óptimos & $4.01-5.00$ \\
\hline Estilos de vida óptimos & $3.01-4.00$ \\
\hline Estilos de vida suficientes para conservar la salud & $2.01-3.00$ \\
\hline Estilos de vida insuficientes para conservar la salud & $<2.01$ \\
\hline
\end{tabular}




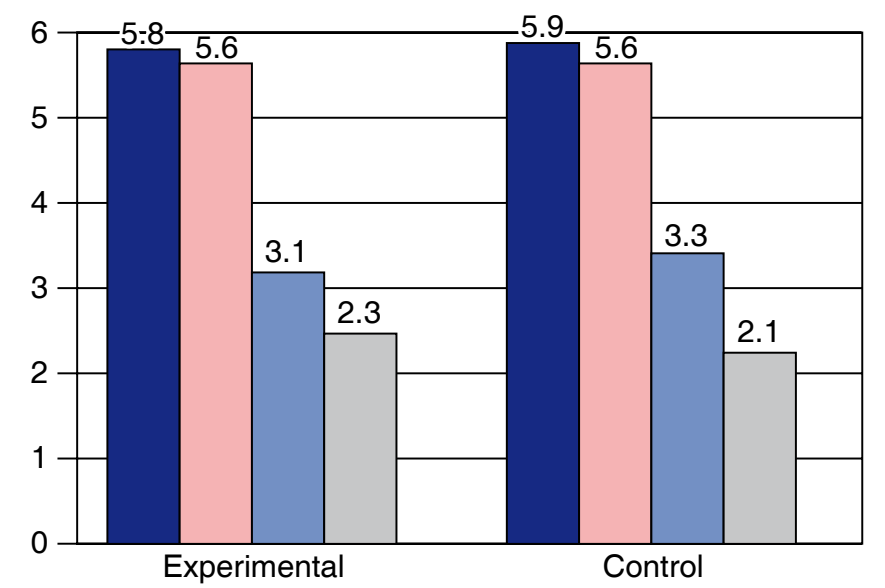

Conocimiento de madres Hábitos de madres

Conocimiento de niños

Habitos de niños

Figura 1. Pre-test, conocimientos y estilos de alimentación saludable.

Respecto al pos-test, sólo entre las madres existieron diferencias significativas en el nivel de conocimientos y actitudes sobre los hábitos alimenticios saludables, haciendo la comparación de los dos grupos: el control y el experimental. Las madres del grupo experimental incrementaron sus conocimientos (8.1 muy óptimos contra 5.9 de las madres del grupo control, que mantuvieron insuficiente su calificación de conocimientos) ( $\mathrm{t}$ $=1.2345$, prob. $=.001$ ), lo que se traduce en un nivel de conocimientos limitados en relación a aspectos nutricionales, el cual se incrementó en madres que tuvieron intervención constante, mientras que estos conocimientos se mantuvieron insuficientes en las madres del grupo control. En cuanto a actitudes sobre estilos de vida, también se observaron diferencias: mientras que las madres del grupo experimental incrementaron sus valores 3.94 (que corresponde a actitudes de nivel óptimo, aunque se mantuvieron en el mismo nivel se elevó su puntaje), las madres del grupo control consiguieron sólo un puntaje de 1.34 (que corresponde a actitudes insuficientes), por lo que se denotan estilos de vida poco saludables respecto a la nutrición ( $\mathrm{t}$ de Student $=1.0672$, prob. $=.001$ ).

En la comparación del pre-test contra el pos-test, existieron diferencias significativas entre el grupo experimental de madres (pre-test y pos-test, $\chi^{2}=.358$, prob. $=.001$ ) aumentando los conocimientos y actitudes en el antes y en el después de la intervención educativa, considerándose como nivel de conocimientos óptimo y actitudes óptimas para conservar la salud.

Es importante notar que en los niños no hubo diferencias significativas por grupo ni en el antes ni en el después.

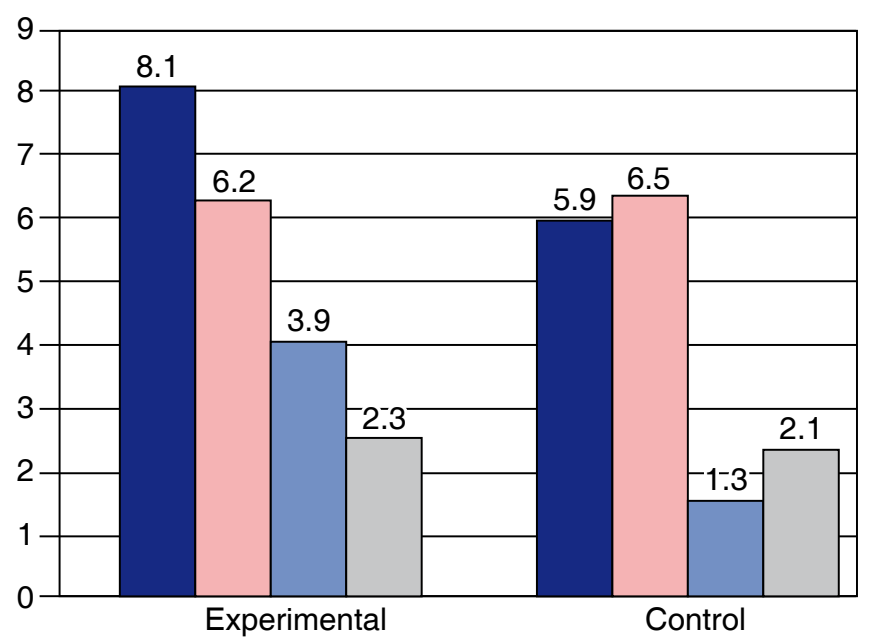

Conocimiento de madres

Hábitos de madres

Conocimiento de niños

Hábitos de niños

Figura 2. Pos-test, conocimientos y estilos de alimentación saludable.

\section{DISCUSIÓN}

Los resultados del estudio coinciden con lo reportado por Ortiz, Rodríguez, Rurangriwa y Pérez, quienes reportaron también que en el grupo donde se ofreció orientación nutricional no se observaron cambios estadísticamente significativos respecto a la reducción de peso, ni en el grupo experimental, ni en el grupo control.

El presente estudio coincide con los datos nacionales reportados y con los encontrados por Briz Hidalgo FJ, Cos Blanco AI y Amate Garrido A, quienes señalan una ligera prevalencia mayor de obesidad en mujeres con relación a los varones.

\section{CONCLUSIONES}

La educación en la nutrición, el interés y práctica de actividades deportivas y/o el ejercicio físico son factores vitales para la salud del escolar; se requiere complementar esfuerzos que induzcan a realizar una actividad física sana y promuevan hábitos alimenticios adecuados, lo cual requiere un trabajo multidisciplinario y la participación activa de la sociedad.

La obesidad en los niños es un asunto de gran preocupación a nivel nacional; cabe señalar que la educación en la nutrición y el deporte son factores vitales para la salud del escolar, pues evitan el desarrollo del sobrepeso y la obesidad. Se requiere de una suma de esfuerzos para lograr estos objetivos.

La familia constituye un factor de apoyo importante para modificar los hábitos de vida sedentarios hacia otros cada 
vez más saludables, fundamentalmente en los niños con problemas de sobrepeso y obesidad. Y aun cuando se han implementado estrategias e intentado diversas intervenciones educativas, como es el caso del presente trabajo, éstas siguen siendo insuficientes para controlar el grave problema de la salud pública.

Aun cuando los niños con sobrepeso u obesidad se benefician con las intervenciones educativas, particularmente con estrategias conductuales y cognitivoconductuales para mejorar la reducción de peso, es claro que hace falta asumir estrategias psicológicas en el niño y la familia que apoyen los cambios hacia los estilos de vida saludables, para así controlar este problema que aumenta cada día.

Es necesario que el profesional de enfermería realice un mayor número de investigaciones e intervenciones para el control de sobrepeso de tipo interdisciplinario, pues el cambiar de hábitos y estilos de vida es un aspecto que requiere tanto un trabajo multidisciplinario como la participación activa de la sociedad.

Debemos tener en cuenta que la educación nutricional, sin la complementación de un cambio en los estilos de vida que incluya actividad física, no conlleva resultados óptimos, reconociendo entonces la necesidad de un trabajo inter y multidisciplinario y la participación activa de la sociedad en general, donde se incluyan actividades interactivas como talleres, para lograr así los cambios de actitud esperados que faciliten estados de salud óptimos.

\section{BIBLIOGRAFÍA}

1. Popkin B. An overview on the nutrition transition and its health implications: The Bellagio meeting. Public Health Nutr 2002; 5 (1A): 93-103.

2. OMS. 10 datos sobre la obesidad [Internet]. 2010 [Consultado el 15 de mayo de 2011]. Disponible en: http://www.who.int/features/factfiles/ obesity/es/

3. OMS. Obesidad y sobrepeso. Nota descriptiva No. 311 [Internet]. Marzo 2011 [Consultado el 22 de abril de 2011]. Disponible en: http://www.who. int/mediacentre/factsheets/fs311/es/

4. ENSANUT [Internet]. Encuesta Nacional de Salud 2006 [Consultado el 23 de julio de 2011]. Disponible en: http://www.insp.mx/ensanut/ ensanut2006.pdf

5. Instituto Nacional de Salud Pública. Encuesta Nacional de Salud y Nutrición 2006. México, D.F. pp. 94-97.

6. Secretaría de Salud. Comunicado de prensa Núm. 236 [Internet]. 27 de abril de 2006 [Consultado el 30 de agosto de 2011]. Disponible en: http://busca.salud.gob.mx/unidades/dgcs/sala_noticias/ comunicados/2006_04_27_236-obesidad_infantil.htm

7. Moraes AS, Beltrán RJ, Mondini L, Freitas MI. Prevalencia de sobrepeso, obesidad y factores asociados en escolares de área urbana. Cad Saúde Pública. Rep Public Health 2004; 22 (6): 1289-1301. En la base de datos LILAC'S Id 428311- Num. 10/79 [acceso el 7 de abril de 2008]. Disponible en: http://bases.bireme.br/cgi-bin/wxislind.exe/iah/online/

8. Ortiz GE, Rodríguez CS, Rurangriwa H, Pérez CP. "Programa multidisciplinario de atención al niño obeso y con sobrepeso" Instituto Mexicano del Seguro Social. Revista de Salud Pública y Nutrición [Internet] 2006 [citada 30 de mayo de 2007]; 4. Disponible en: http://www.respyn.uanl. $\mathrm{mx} /$ especiales/2006/ee-042006/documentos/nutricion.htm

9. Moraga MF, Rebollo GMJ, Bórquez VP, Cáceres DJ, Castillo DC. Tratamiento de la obesidad infantil: Factores pronósticos asociados a una respuesta favorable. Rev Chil Pediatr [Internet] 2003 [citado 3 octubre 2011]; 74 (4): 374-380. Disponible en: http://www.scielo.cl/scielo. php?script=sci_arttext\&pid=S0370-41062003000400004\&lng=es

10. Briz HFJ, Cos BAI, Amate GAM. Prevalencia de obesidad infantil en Ceuta. Estudio PONCE 2005. Nutr Hosp 2007; 22 (4): 471-77. 